\title{
Sistemas Híbridos para Recomendações Educacionais: Uma Revisão Sistemática da Literatura
}

\author{
Thayron Crystian Hortences de Moraes $^{1,2}$ Itana $_{\text {Stiubiener }}^{1}$ \\ ${ }^{1}$ Programa de Pós-Graduação em Ciência da Computação \\ Universidade Federal do ABC (UFABC) \\ Santo André - SP - Brazil \\ ${ }^{2}$ Faculdade de Engenharia (FAEng) \\ Universidade Federal de Mato Grosso (UFMT) \\ Várzea Grande - MT - Brazil \\ \{thayron.moraes, itana.stiubiener\} @ufabc.edu.br
}

\begin{abstract}
This paper presents the results of a Systematic Review of Literature on Educational Recommendation System, specifically on how hybrid approaches are being used in this context, we searched for papers in six scientific databases, obeying the following research question: "Which are the hybrid approaches proposed in the literature for recommending educational resources?". Following a defined methodology, we found 67 studies of which several information were extracted as: most used techniques, number of publications per year and most cited works.
\end{abstract}

Resumo. Este artigo apresenta os resultados de uma Revisão Sistemática da Literatura sobre Sistema de Recomendação Educacionais, especificamente sobre como abordagens híbridas estão sendo utilizadas nesse contexto. Para tanto, buscou-se artigos em seis bases de dados científicos, obedecendo a seguinte questão de pesquisa: "Quais são as abordagens híbridas propostas na literatura para recomendação de recursos educacionais?”. Seguindo uma metodologia definida, encontrou-se 67 estudos dos quais foram extraídas várias informações como: técnicas mais utilizadas, número de publicações por ano e trabalhos mais citados.

\section{Introdução}

Escolher e selecionar recursos educacionais pode ser um processo complicado face a grande disponibilidade destes recursos, e pode não trazer os resultados esperados. Os Sistemas de Recomendação Educacionais (SRE) surgiram para enfrentar este problema.

Os Sistemas de Recomendação (SR) típicos, ou seja, comerciais, são sistemas inteligentes que buscam e oferecem itens com maior probabilidade de interesse para um usuário específico (RICCI; ROKACH; SHAPIRA, 2015); Enquanto os SRE, visam adaptar e personalizar o processo de ensino-aprendizagem, tal abordagem visa recomendar aos aprendizes Objetos de Aprendizagem (OAs) apropriados ao seu perfil de aprendizagem (AGUIAR et al., 2017) adaptando o sistema as suas experiências, visando suprir a alta diversidade e aumentando sua satisfatibilidade (GULZAR; LEEMA, 2018). 
VIII Congresso Brasileiro de Informática na Educação (CBIE 2019)

Anais do XXX Simpósio Brasileiro de Informática na Educação (SBIE 2019)

A palavra perfil pode ser considerada como Estilo de Aprendizagem (EA), importante para entender como um aprendiz compreende e participa de atividades de aprendizagem. Servindo para determinar como aprendizes interagem e reagem, refletindo suas características individuais referentes às tarefas de organizar, perceber, processar, lembrar e pensar para resolver um problema, tornando possível ao SRE fornecer conteúdos relevantes ao aprendiz (ZAINA et al., 2012).

Tarus, Niu e Mustafa (2018) em sua pesquisa de revisão aborda algumas das principais técnicas de recomendação utilizadas em SRs na atualidade, conforme:

- Filtragem Colaborativa $(\boldsymbol{C F})$ : O princípio está na troca de experiências entre as pessoas que possuem interesses comuns, considerando avaliações passadas de usuários com gostos semelhantes para recomendar itens;

- Baseada em Conteúdo (CB): Relaciona descrições dos conteúdos dos itens com as preferências dos usuários (classificações, visitas, etc.), visando verificar se o item é ou não relevante;

- Baseada em Conhecimento (KB): Usa as preferências do usuário e suas necessidades para realizar recomendações através do conhecimento que se possui das relações de um item para com um determinado usuário;

- Baseada em Ontologia (OB): São SR baseados em conhecimento (KB) que usam ontologia como representação do conhecimento;

- Baseada em Demografia (DB): Utiliza o perfil demográfico para aprender o relacionamento entre um item em particular e o tipo de usuário que poderia vir a se interessar;

- Baseada em Utilidade (UB): Realiza um cálculo da utilidade de cada objeto para o usuário. Tais cálculos podem ser baseados nas preferências do usuário;

- Sensível ao Contexto (CA): Informações contextuais são usadas para filtrar dados antes de aplicar um algoritmo tradicional de recomendação. Neste sentido, o contexto é definido como qualquer informação que possa ser usada para caracterizar a situação de uma entidade!

- Baseada em Confiança (TA): O objetivo é gerar recomendações personalizadas a partir de opiniões e associações conhecidas, cria um critério de confiança e compromisso assumindo que ações futuras levarão a um bom resultado;

- Baseada em Redes Sociais (SB): Esses SRs geram recomendações com base em informações sobre perfis de usuários e relacionamentos entre usuários;

- Baseada em Lógica Fuzzy (FB): São geradas recomendações com base em informações incertas ou vagas. Por exemplo, em muitas situações práticas, os usuários expressam sua preferência por itens em termos linguísticos tais como "muito interessado" ou "não interessado";

- Baseada em Grupos (GB): Os SR de grupo recomendam itens para um grupo de usuários e não para um indivíduo, tipo de técnica adequada para situações em que várias pessoas participam de uma única atividade.

- Híbrida: Relaciona mais de uma técnica de filtragem simultaneamente, superando as limitações de cada modalidade e permitindo um resultado mais preciso.

SRs híbridos buscam enriquecer a qualidade da recomendação e superar limitações, em SRE abordagens híbridas relacionam técnicas de recomendação com teorias educacionais e métodos computacionais na busca de resultado que sejam relevantes ao processo de ensino-aprendizagem. Desta forma, este artigo tem como objetivo estudar o estado da arte sobre SRE híbridos por intermédio de uma Revisão Sistemática da Literatura (RSL).

\footnotetext{
${ }^{1}$ Entidade pode ser considerada uma pessoa, local ou objeto relevante para a interação entre um usuário e um aplicativo, incluindo o usuário e os próprios aplicativos.
} 
VIII Congresso Brasileiro de Informática na Educação (CBIE 2019)

Anais do XXX Simpósio Brasileiro de Informática na Educação (SBIE 2019)

\section{Trabalhos Relacionados}

Ao examinar a literatura, é possível encontrar inúmeros estudos que buscam analisar SRE. Em Aguiar et al. (2015) é realizado um Mapeamento Sistemático (MS) da literatura com o objetivo de identificar e analisar a produção científica referente a SRE no Brasil, assim como Ferreira, Vasconcelos e França (2017). Em Pontes et al. (2014) é realizada uma RSL sobre filtragens de recomendação de Objetos de Aprendizagens publicados nos anais do CBIE nos anos de 2008 à 2013.

Nascimento et al. (2017) faz uma RSL buscando avaliar o estado da arte sobre a recomendação de objetos de aprendizagem baseadas em EA entre os anos de 2002 à 2016. Já Rolim et al. (2017) trás um estudo acerca dos sistemas de recomendação de recursos educacionais trazendo um panorama entre 2008 e 2017. Desta forma, está pesquisa busca contribuir com uma análise sobre a recomendações híbridas para recursos educacionais.

\section{Procedimentos Metodológicos}

Esta Revisão Sistemática da Literatura (RSL) foi baseada no trabalho proposto por Kitchenham et al. (2010), que favorece a identificação, avaliação e análise de estudos significativos que permitem identificar, avaliar e interpretar pesquisas disponíveis e relevantes para uma determinada questão de pesquisa ou área temática levantando/investigando o estado da arte. A pesquisa foi realizada com o auxílio da ferramenta StArt (State of the Art through Systematic Reviews) na versão 3.3 (Beta) desenvolvida pelo Laboratório de Engenharia de Software (LAPES) da Universidade Federal de São Carlos (UFSCAR).

\subsection{Questões de Pesquisa}

Buscando ampliar e contribuir com trabalhos na área de recomendação de recursos educacionais, uma vez que, SRE utilizam-se de diferentes técnicas, estratégias e métodos, buscando analisar a recomendação destes recursos à aprendizes, e, principalmente, atender as suas características e necessidades, devem ser elaborados problemas de pesquisa sólidos.

Neste sentido, e, para delinear o escopo e direcionar o desenvolvimento deste trabalho, as questões de pesquisa foram elaboradas com base na estratégia Population, Intervention, Comparison e Outcome (PICO), sugerida por Biolchini et al. (2007). Seguindo essa estratégia, a RSL tem como questão principal “QP1 - Quais são as abordagens híbridas propostas na literatura para recomendação de recursos educacionais?" sendo a motivação obter o estado da arte sobre abordagens híbridas de recomendação no contexto educacional.

\subsection{Planejamento da RSL}

Foi realizada uma busca por trabalhos publicados entre 2002 e $2018^{2}$, e disponibilizados em periódicos ou anais de eventos online de relevância, tais como: ACM Digital Library ${ }^{3}$, Portal de Publicações da Comissão Especial de Informática na Educação (CEIE) ${ }^{4}$ IEEE Xplore

\footnotetext{
${ }^{2}$ Considera-se este período uma vez que, o trabalho publicado por Burke (2002) é considerado um marco para SR híbridos.

${ }^{3}$ https://dl.acm.org/

${ }^{4}$ http://www.br-ie.org/pub/
} 
Digital Library $\bigsqcup_{5}^{5}$ Science Direct $t^{6}$ Scopus $7^{7}$ e a Springer $\rrbracket^{8}$, sendo estas consideradas fontes que possuem um amplo conjunto de publicações relacionadas à Informática e Educação.

\subsubsection{Expressão de Busca}

É possível analisar os resultados obtidos a cada execução da string nas bases de busca, incluir ou remover palavras para que os resultados estejam alinhados aos objetivos da pesquisa, os trabalhos devem conter os termos da string no título, resumo ou palavraschave. As opções de filtragem fornecidas pelas bases de dados também integraram o processo da busca, foram selecionados: o ano inicial das publicações desejadas e o idioma de escrita dos trabalhos.

Para a elaboração da expressão de busca, utilizou-se palavras-chave que representam a Population e Intervention, sendo constituídas por palavras relacionadas a hybrid recommender, learning objects e educational resources, respectivamente, conforme apresentado abaixo:

(hybrid recommend*) AND ((learn* OR education*) AND (resources OR objects))

\subsubsection{Critérios de Inclusão e Exclusão}

Os critérios de inclusão utilizados foram: (i) Estudos publicados a partir do ano de 2002; (ii) Pesquisas, artigos de conferências e journals; (iii) Estudos que relatem abordagens híbridas para SRE. E os critérios de exclusão foram: (i) Artigos com menos de três páginas; (ii) Estudos anteriores a 2002; (iii) Documentos repetidos ou redundantes; (iv) Versão anterior de um documento; (v) Estudos que não focam em SRE híbridos; (vi) Documento pago ou que não estejam disponíveis para leitura; (vii) Documento em idioma diferente do inglês, português ou espanhol.

\subsubsection{Qualificação dos Trabalhos}

A qualificação dos trabalhos ocorreu de acordo com um questionário, em que foram informadas respostas "sim", "não" ou "parcialmente". As questões aplicadas serviram como indicador de qualidade e relevância de cada trabalho na RSL, conforme:

QQ1 Os objetivos estão claramente definidos?

QQ2 As abordagens de recomendação estão claramente definidas?

QQ3 Ficou claro os critérios de escolha das técnicas de recomendação?

QQ4 Foi realizado experimentos e os resultados foram comparados com outros?

QQ5 Foram apresentadas e/ou discutidas limitações ou pontos negativos?

QQ6 A abordagem se mostrou útil para recomendar recursos educacionais?

\footnotetext{
${ }^{5}$ http://ieeexplore.ieee.org/

${ }^{6} \mathrm{https}: / / \mathrm{www}$. sciencedirect.com/

${ }^{7}$ https://www.scopus.com/home.uri

${ }^{8} \mathrm{https}: / /$ www.springeropen.com/
} 
VIII Congresso Brasileiro de Informática na Educação (CBIE 2019)

Anais do XXX Simpósio Brasileiro de Informática na Educação (SBIE 2019)

\section{Condução da RSL}

A busca nas fontes de pesquisa e a seleção das publicações de acordo com os critérios de inclusão e exclusão, bem como a avaliação de qualidade, ocorreram entre Outubro de 2018 a Janeiro de 2019. O processo de seleção dos trabalhos foi divido em quatro fases e cada fase consistia em um tipo de verificação e análise que foi aplicada em cada trabalho, a Tabela 1 mostra a quantidade de artigos obtidos em cada fase do processo.

Tabela 1. Quantidade de estudos encontrados em cada fase

Fonte de Pesquisa Fase 01 Fase 02 Fase 03 Fase 04

\begin{tabular}{ccccc} 
ACM Digital Library & 894 & 852 & 15 & 04 \\
CEIE & 77 & 74 & 17 & 09 \\
IEEE Xplore & 65 & 57 & 16 & 07 \\
Science Direct & 88 & 81 & 16 & 07 \\
Scopus & 313 & 226 & 49 & 28 \\
Springer & 314 & 235 & 18 & 04 \\
\hline Total & 1751 & 1525 & 131 & 67 \\
\hline
\end{tabular}

O processo de seleção foi realizado conforme descrito nas fases a seguir:

Fase 1 Busca nas bases de dados com a utilização da string;

Fase 2 Aplicada a (Filtragem 1) - São desconsiderados os trabalhos duplicados e os que não estavam escritos em inglês, português ou espanhol e anteriores a 2002;

Fase 3 Aplicada a (Filtragem 2) - Passam a ser lidos os títulos, resumos, e, conforme os critérios de inclusão e exclusão, os trabalhos são selecionados para eliminar aqueles trabalhos que não têm como foco no objetivo desta pesquisa, ou seja, SRE híbridos;

Fase 4 Aplicada a (Filtragem 3) - É realizada uma leitura superficial dos artigos avaliando conforme critérios de qualidade; e, observando os critérios de inclusão e exclusão em outras seções do artigo.

Os artigos selecionados de cada base de dados, após a (Filtragem 3), podem ser vistos no Anexo A.

\subsection{Qualificação de Dados}

Conforme é descrito por Kitchenham et al. (2010), a qualificação dos trabalhos deve seguir um processo de complementação dos critérios de inclusão e exclusão, onde são atribuídos pesos, Não $=0$, Parcialmente $=0.5$ e $\operatorname{Sim}=1$, esse processo ajuda a investigar se há diferentes explicações para os resultados dos trabalhos e uma forma de ponderação individual dos trabalhos durante a sintetização dos resultados, os valores quantitativos do questionário de avaliação de qualidade é apresentado na Tabela 2. 
VIII Congresso Brasileiro de Informática na Educação (CBIE 2019)

Anais do XXX Simpósio Brasileiro de Informática na Educação (SBIE 2019)

Tabela 2. Questionário de Avaliação de Qualidade

\begin{tabular}{lccc} 
ID & Sim & Parcial & Não \\
\hline QQ1 & $67(100 \%)$ & $0(0 \%)$ & $0(0 \%)$ \\
QQ2 & $60(90 \%)$ & $7(10 \%)$ & $0(0 \%)$ \\
QQ3 & $25(37 \%)$ & $36(54 \%)$ & $6(9 \%)$ \\
QQ4 & $11(16 \%)$ & $38(57 \%)$ & $18(27 \%)$ \\
QQ5 & $9(13 \%)$ & $4(6 \%)$ & $54(81 \%)$ \\
QQ6 & $27(40 \%)$ & $34(51 \%)$ & $6(9 \%)$ \\
\hline
\end{tabular}

Conforme as questões apresentadas anteriormente podemos considerar:

QQ1 foi proposta para verificar se o trabalho tem potencial para ser inserido nessa RSL, trabalhos marcados com "sim" serão analisados e os que obtiverem resposta "não" serão excluídos. A quantidade de "sim" foi de $100 \%$;

QQ2 caracteriza os trabalhos que apresentaram em detalhes as abordagens de recomendação utilizadas, sendo 90\% de "sim" e 10\% "parcial", percebe-se que em alguns trabalhos não ficou totalmente claro o que foi utilizado ou proposto;

QQ3 foi proposta para refletir sobre os critérios de escolha das técnicas utilizadas nas abordagens, os trabalhos categorizados como "sim" apontam aqueles onde foram apresentados os critérios e foram expostos os motivos para o contexto educacional, sendo 37\% das respostas; as marcadas como "parcial" apontam aqueles onde foram apresentados os critérios nem contexto técnico e/ou tendencias e "não" onde as técnicas são decorrentes de trabalhos anteriores ou não foram apresentados os motivos, representando $54 \%$ e $9 \%$ do total, respectivamente;

QQ4 descreve os trabalhos onde houve avaliações experimentais, sendo categorizado os trabalhos assinalados com "sim" aqueles onde foi realizada avaliação experimental (quantitativa e/ou qualitativa) e os resultados foram comparados com outros trabalhos, representando 16\%; "parcial" aponta aqueles onde foram realizadas avaliação experimental (quantitativa e/ou qualitativa), mas não houve comparação com resultados de outros autores, representando 57\%; já, "não" para trabalhos onde não foram realizadas avaliações experimentais;

QQ5 mostram que a maior parte dos autores não discutem nos artigo os resultados que não foram considerados bons ou as limitações encontradas nos processos, entretanto, trabalhos assinalados com "sim" apontam aqueles onde foram discutidas/apresentas limitações, inclusive para o contexto educacional e "parcial" onde somente houve a apresentação, mas não a discussão para o contexto educacional;

QQ6 caracteriza os trabalhos representados por "sim" apontam trabalhos onde as abordagens se mostraram uteis tendo como base avaliações experimenteis (quantitativa e qualitativa), representando 40\%; "parcial" aponta aqueles onde as abordagens se mostraram uteis tendo como base avaliações experimenteis (quantitativa e/ou qualitativa), representando 40\%; e, "não" para trabalhos onde somente é apresentada a abordagem e não foram realizadas avaliações experimentais para verificar sua efetividade. 
VIII Congresso Brasileiro de Informática na Educação (CBIE 2019)

Anais do XXX Simpósio Brasileiro de Informática na Educação (SBIE 2019)

\section{Análise dos Dados}

A partir dos trabalhos selecionados foi realizada uma extração de informações, a avaliação da qualidade dos trabalho cooperou para enriquecer as conclusões e para a coerência da síntese. Os 67 artigos selecionados (ver Anexo B) que atenderam aos critérios de inclusão e exclusão, apresentaram abordagens de recomendação híbridas para o contexto educacional. Dos resultados obtidos, apresentados na Tabela 1, foram extraídos os anos. Podemos verificar na Figura 1 a quantidade de artigos publicados em cada ano.

Figura 1. Publicações por ano

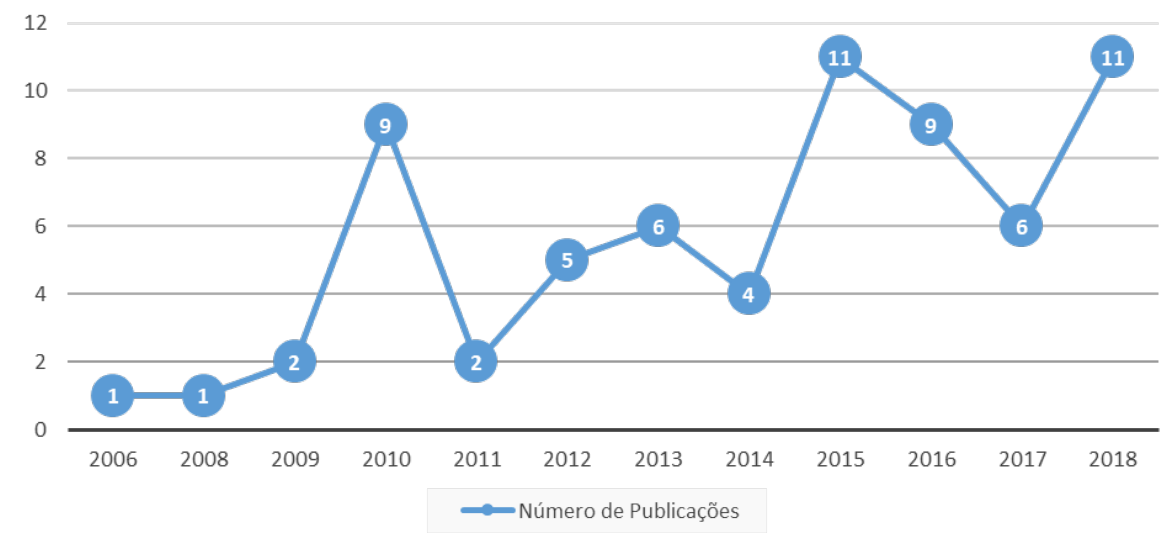

\subsection{QP1: Quais as abordagens híbridas propostas na literatura para recomendação de recursos educacionais?}

Com relação às técnicas de recomendação utilizadas nas abordagens híbridas estudadas, podemos verificar que a técnica mais utilizada é a $\mathrm{CF}$, esta associada a outras técnicas ou métodos.

Consideramos, assim, que o percentual de presença de cada técnica nas abordagens estudadas são: Outras (73\%); CF (57\%); CB (37\%); OB (25\%); CA (15\%); KB (13\%); FB (9\%); GB (4\%); UB (3\%); TA (3\%); DB (1\%); SB (1\%); Destaca-se entre outros métodos a utilização da Mineração de Dados.

Podemos perceber que o total de métodos computacionais utilizados nas abordagens híbridas, em sua grande maioria é de 55\%, as abordagens apresentadas utilizam-se de outros mecanismos, associados ou não às técnicas de recomendação mais conhecidas.

A taxa dos trabalhos que realizaram testes e/ou avaliações para validação e qualificação das abordagens propostas são:

(i) em, $25 \%$ dos trabalhos não foi realizada nenhuma experimentação;

(ii) em, $75 \%$ foram realizados experimentação, destas:

(a) em, 11\% foram realizadas uma experimentação com dados de aprendizes obtidos de maneira online, tais dados foram comparados com outras abordagens para comparação de eficiência e satisfação das recomendações, sendo então caracterizado como misto;

(b) em, $36 \%$ as experimentações foram realizadas em ambientes online com uma amostragem de aprendizes, buscando-se principalmente analisar a satisfação dos aprendizes com as recomendações; 
VIII Congresso Brasileiro de Informática na Educação (CBIE 2019)

Anais do XXX Simpósio Brasileiro de Informática na Educação (SBIE 2019)

(c) em, 28\% para as experimentações foram utilizados com conjuntos de dados obtidos de fontes externas ou já coletados de AVA previamente utilizados, caracterizados de offline, nestes, o objetivo principal foi comparar a qualidade das recomendações entre diferentes abordagens.

Dentre os procedimentos realizados para as experimentações podemos destacar o uso de: (i) Casos de Estudo; (ii) Questionários; (iii) Provas de Conceito; (iv) testes de Precisão; (v) uso de Mean Absolute Error (MAE); (vi) testes de Performance; (vii) Recall (abrangência); (viii) métrica F1 Score; e, (iv) testes de Esparsidade.

\section{Limitações da RSL}

Esta RSL foi elaborada com base em um protocolo robusto, entretanto seu processo é sujeito a vulnerabilidades. Tais limitações podem ser:

(i) Exclusão de algum trabalho relevante, que pode não ter correspondido aos critérios de inclusão definidos no protocolo (ver Subsubseção 3.2.2);

(ii) Decisões subjetivas do revisor, em razão da, exclusão de trabalhos que não apresentaram uma descrição clara;

Contudo, utilizou-se um método robusto e pesquisa nas principais bases de publicação sobre Informática na Educação a fim de obter um panorama sobre abordagens híbridas para SRE, mostrando significativos resultados.

\section{Discussão dos Resultados e Conclusões}

Neste artigo foi apresentada uma revisão sistemática da literatura (RSL) sobre Sistemas de Recomendação Educacionais (SRE), especificamente em como abordagens híbridas estão sendo utilizadas para recomendar recursos educacionais. Diversas abordagens híbridas para recursos educacionais foram encontradas na literatura e passaram por algumas etapas de análise e classificação, para responder a questão de pesquisa propostas nesta RSL.

Desta forma identificou-se através desta pesquisa os seguintes pontos:

(i) Grande parte das pesquisas selecionadas tratam da recomendação de OAs (60\%), o restante dos trabalhos apresentam abordagens para recomendação de recursos de aprendizagem (30\%) que podem ser (cursos online, caminhos de aprendizagem, padrão pedagógico, etc...) e 10\% para Outros recursos.

(ii) Ficou clara o crescimento da preocupação com as necessidades cognitivas do aprendiz, podemos perceber que dos 67 trabalhos selecionados $31 \%$ utilizaram EA em suas abordagens, entretanto a maioria (69\%) não utilizou ou descreveu o uso de EA em sua abordagem, neste sentido na Figura 2 podemos ver a progressão em que o EA é aplicado nos trabalhos selecionados; 
Figura 2. EA por Ano

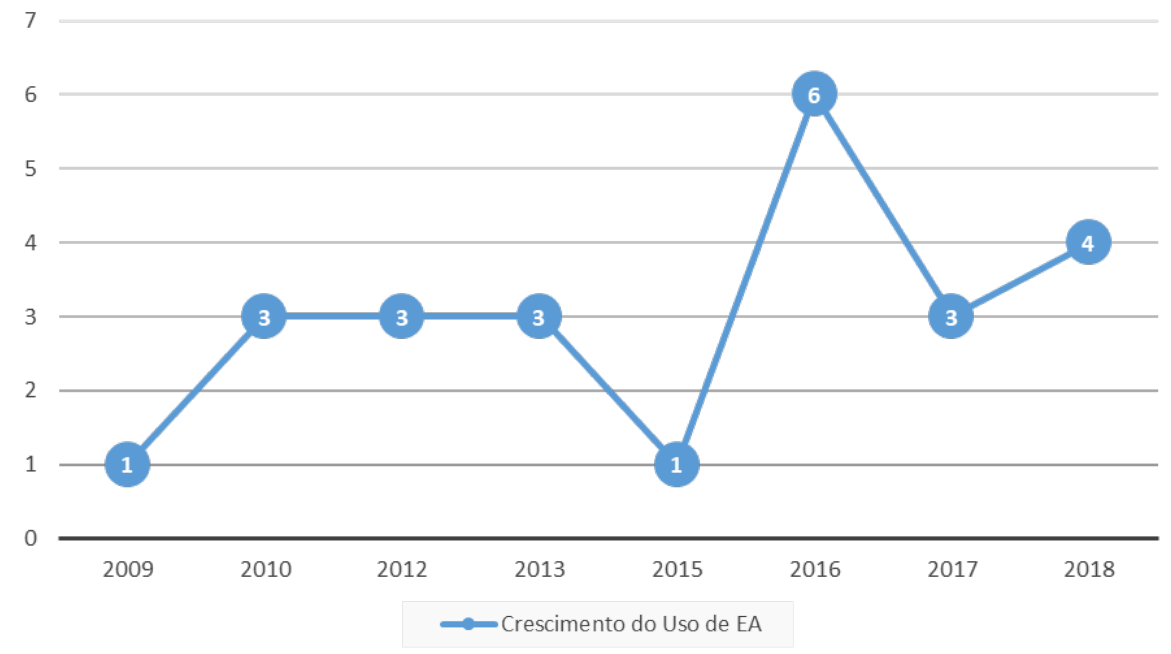

(iii) Percebeu-se que em grande parte das abordagens o padrão de metadados utilizado foi o LOM (27\%), apesar de grande parte dos trabalhos (69\%), não especificar o padrão empregado;

(iv) Técnica $\mathrm{CF}$ - Ainda que a $\mathrm{CF}$ seja a técnica mais associada às abordagens híbridas, aparecendo em 57\% dos trabalhos selecionados, não existe um consenso sobre como o modelo deve ser aplicado, sendo as heurísticas mais populares baseadas na correlação de Pearson, distância (euclidiana e Manhattan), e na função cosseno. Peralta et al. (2017) discute sobre limitações destas técnicas e apresenta o método de Regressão de Tópico Colaborativo (CTR) de última geração que utilizou um modelo gráfico probabilístico que integra perfeitamente um modelo de tópico, a alocação latente de Dirichlet (LDA) e um método de CF baseado em modelo, a fatoração de matriz probabilística (PMF), que por meio de testes se mostrou mais eficiente;

(v) Percebeu-se ainda que além de técnicas de filtragem conhecidas a utilização de outros métodos computacionais como Mineração Dados e Redes Neurais;

Podemos concluir com esta RSL com o objetivo de personalizar o ensino SRE vem crescendo, bem como a utilização de abordagens híbridas. Entretanto definir qual abordagem utilizar para realização da recomendação de recursos educacionais, requer conhecimento das técnicas de recomendação e habilidade para compreender as necessidades do ambiente e dos aprendizes. Tais necessidades, refletem a preocupação com o processo de ensino-aprendizagem do aprendiz, que por vezes, levam em consideração suas preferências, interesses e sua trajetória, nestes sistemas podem ser usados diversos modelos, sendo um deles o EA de Felder e Soloman (1991).

Espera-se através desta RSL evidenciar trabalhos de pesquisa no contexto dos SRE que realizam recomendações por dentro de abordagens híbridas, contribuindo assim para a área. 
VIII Congresso Brasileiro de Informática na Educação (CBIE 2019)

Anais do XXX Simpósio Brasileiro de Informática na Educação (SBIE 2019)

\section{Referências}

AGUIAR, J. et al. Um Estudo sobre a Influência das Dimensões do Modelo FelderSilverman na Recomendação de Recursos Educacionais baseada nos Estilos de Aprendizagem dos Alunos. In: 1. BRAZILIAN Symposium on Computers in Education (Simpósio Brasileiro de Informática na Educação-SBIE). 2017. v. 28, p. 1277.

AGUIAR, J. et al. Avaliação de Sistemas de Recomendação Educacionais no Brasil: uma revisão sistemática da literatura. In: 1. BRAZILIAN Symposium on Computers in Education (Simpósio Brasileiro de Informática na Educação-SBIE). 2015. v. 26, p. 1255. BIOLCHINI, J. C. d. A. et al. Scientific research ontology to support systematic review in software engineering. Advanced Engineering Informatics, Elsevier, v. 21, n. 2, 2007. BURKE, R. Hybrid recommender systems: Survey and experiments. User modeling and user-adapted interaction, Springer, v. 12, n. 4, p. 331-370, 2002.

FELDER, R. M.; SOLOMAN, B. A. Index of learning styles. 1991. Disponível em: <wWw.ncsu.edu/effective_teaching/ILSdir/ILS-a.htm>.

FERREIRA, V.; VASCONCELOS, G.; FRANÇA, R. Mapeamento Sistemático sobre Sistemas de Recomendações Educacionais. In: 1. BRAZILIAN Symposium on Computers in Education (Simpósio Brasileiro de Informática na Educação-SBIE). 2017. v. 28, p. 253. GULZAR, Z.; LEEMA, A. A. A Framework for Recommender System to Support Personalization in an E-Learning System. International Journal of Web-Based Learning and Teaching Technologies (IJWLTT), IGI Global, v. 13, n. 3, p. 51-68, 2018.

KITCHENHAM, B. et al. Systematic literature reviews in software engineering-a tertiary study. Information and software technology, Elsevier, v. 52, n. 8, p. 792-805, 2010.

NASCIMENTO, P. et al. Recomendação de Objetos de Aprendizagem baseada em Modelos de Estilos de Aprendizagem: Uma Revisão Sistemática da Literatura. In: 1. BRAZILIAN Symposium on Computers in Education (Simpósio Brasileiro de Informática na EducaçãoSBIE). 2017.

PERALTA, M. et al. Understanding Learning Resources Metadata for Primary and Secondary Education. IEEE Transactions on Learning Technologies, IEEE, 2017.

PONTES, W. L. et al. Filtragens de Recomendação de Objetos de Aprendizagem: uma revisão sistemática do CBIE. In: 1. BRAZILIAN Symposium on Computers in Education (Simpósio Brasileiro de Informática na Educação-SBIE). 2014. v. 25, p. 549.

RICCI, F.; ROKACH, L.; SHAPIRA, B. Recommender systems: introduction and challenges. In: RECOMMENDER systems handbook. Springer, 2015. p. 1-34.

ROLIM, V. et al. Um Estudo Sobre Sistemas de Recomendação de Recursos Educacionais. In: 1. ANAIS dos Workshops do Congresso Brasileiro de Informática na Educação. 2017. TARUS, J. K.; NIU, Z.; MUSTAFA, G. Knowledge-based recommendation: a review of ontology-based recommender systems for e-learning. Artificial Intelligence Review, Springer, v. 50, n. 1, p. 21-48, 2018.

ZAINA, L. et al. E-LORS: Uma abordagem para recomendação de objetos de aprendizagem. Revista Brasileira de Informática na Educação, v. 20, n. 1, p. 04, 2012. 